\title{
PRIMO MILLENARIO DELLA LINGUA ITALIANA
}

(960-1960)

\author{
"LA CARTA DI CAPUA"
}

\section{Carolina Albanese}

(da Universidade Católica de Curitiba e da "Dante Alighieri")

Capua, città della Campania, sorge in un'ansa del fiume Volturno.

Cli oliveti ben piantati ad uguale distanza, il granturco con la sua scarmigliata capigliatura, i vigneti sparsi dappertutto, a una distesa che non sempre lo sguardo raggiunge, danno alla città un aspetto ridente, il brillare d'un riso di natura viva.

Ed in mezzo a questo sereno orizzonte emergono fastigi di chiese, strade ampie, palazzi, testimonianze di un passato glorioso intercalato da luci ed ombre.

Capua antica era situata a pochi chilometro dall'attuale e la sua fondazione risale, probabilmente, al 598 a. C.

Varie dominazioni vi si succedettero, prima fra tutte la sannita e la romana.

Nel 340 circa, per timore dei Sanniti, Capua si alleò ai Romani. Col passare del tempo, detta lega si fece gravosa così che i Campani, approfittando del disastro di Canne, si ribellarono a Roma e giurarono amicizia ai Cartaginesi.

In Capua si accampò Annibalé, timoroso di assaltare Roma. Colà, il celebre generale cartaginese si abbandonò ai "famosi ozi", che lo snervarono e lo condussero a chiedere pace al popolo romano.

Non sorte migliore toccò a Capua, che si arrese nel 211. 11 territorio fu confiscato per più di mezzo secolo, fino a quando. nel 61, Cesare vi institui una colonia, ridando vita alla città. 
Nonostante le devastazioni, tra cui è famosa quella operata da Gianserico nel 465 d. C., Capua continuò a prosperare, tanto che Paolo Diacono, nel VII secolo, l'indica fra le tre principali città della Campania.

Dei resti archeologici, bellissimi sono l'anfiteatro, edificato da Augusto e ornato di statue da Adriano, e un arco di mattoni a tre fornici.

Quest'anno Capua attrae particolarmente l'attenzione dei glottologi, filologi studiosi in genere, nonchè degl'Italiani tutti. Viene difatti celebrato il millenario della "Carta di Capua" (9601960).

Si tratta di un placito del 960 , concernente i beni di un monastero dipendente da Montecassino. $\dot{E}$, in breve, una sentenza che pone termine alla controversia sorta tra gli abati ed un tale Rodelgrimo, circa il possesso di alcune terre rivendicate da entrambe le parti. Contenuto pertanto comunissimo, rintracciabile in documenti di qualsiasi epoca.

La "Carta di Capua" è scritta in latino; ma essa contiene pure le deposizioni di alcuni testimoni, fatte in lingua volgare. Da qui l'importanza della "Carta di Capua".

Il passo in volgare è il seguente:

- Sao ko kelle terre, per kelle fini que ki contene, trenta anni le possette parte sancti Benedicti -.

Occorre tener presente che, a quei tempi, la conoscenza del latino era base essenziale alla cultura. Epigrafi, carte pubbliche e private, trattati filosofici e giuridici, poemi, cronache etc., erano scritti esclusivamente in latino.

Per la prima volta, quindi, con la "Carta di Capua", il volgare è usato volutamente in un atto pubblico. Ciò dimonstra che, in quell'epoca, il popolo parlava una lingua che non era piú precisamente il latino.

"Volutamente" in quanto, i testimoni, chierici e notai, sarebbero stati atti ad $\epsilon$ mettere in latino le loro testimonianze. Essi vollero, di certo, pronunziare le "formole" in volgare affinchè il pubblico le comprendesse. 
La forma sao usata nella "Carta di Capua" ed in quelle successive (Carta di Sessa, marzo 963; Carta di Teano, ottobre 963), ha dato adito a diverse interpretazioni.

Secondo il glottolcgo Bartoli, sao proviene da un'area linguistica settentrionale. "Le formule cassinesi rispecchiano un linguaggio regionale, della Campania, quale era parlato abitualmente dai giureconsulti e dagli ecclesiastici campani della seconda metà del sec. $X$. Ma quel linguaggio regionale conteneva anche elementi interresionali e di due specie diverse: latinismi e italianismi. O meglio e più semplicemente: elementi latini e italiani. Il più sicuro di tali elementi è sao, onde so" (1).

Dall'ipotesi del Bartoli, discorda il letterato Bruno Migliorini e con lui molti altri, ritenendo sao una formazione analogica scaturita dalle forme di 2a. e 3a. persona, "sai" (lat. sapis), e "sae" (lat. sapit), e da presenti come ao, stao etc., incontrati nei dialetti campani verso il mille. Successivamente, sempre secondo il Migliorini ed altri, Capua subi l'influenza del meridionale "saccio" o "sazzo" o "sacce", continuazione del latino "sapio" (2).

Comunque non ci soffermeremo oltre sull'analisi delle forme per non incorrere in eccessive deviazioni. II nostro scopo è infatti illustrare l'importanza della "Carta di Capua", o meglio, il suo valore come documentazione ed affermazione scritta del volgare.

E chiaro che l'italiano non è nato nel marzo del 960 , ed assurdo sarebbe il pretendere di segnarne la data di nascita in quanto esso non è lingua diversa dal latino né tampoco la contrapposizione. Como ben dice il Sansone, nella sua Storia della Letteratura Italiana, "L'italiano è la stessa lingua latina in un suo particolare modo e momento di svolgimento".

L'Aquila romana, nella sua espansione territoriale, evitava di dare alle terre conquistate il carattere rigido d'una colonizza-

(1) In Lingua Nostra, VI. 1944-45, pp. 1-6.

(2) Cf. Bruno Migliorini. Storia della Lingua italiana, Sansoni, Firenze, 1960. D. 95 . 
zione militare. Si instauravano rapporti civili, commerciali, culturali, di modo che i popoli soggiogati vedevano in Roma, non la dominatrice spietata, bensì il fulcro della civiltà.

E ne subirono il fascino a tal punto da sostituire l'originario idioma con quello romano.

Logicamente non risultò un vero e proprio latino letterario, perchè in genere i nativi imparavano detta lingua a contatto con i soldati e $i$ coloni. Fatto è che il latino penetrò in nuovi domini, e di conseguenza, tra nuove genti.

Si sa che la lingua è un qualcosa di vivo che continuamente si evolve e trasforma, per cui anche il latino era destinato fatalmente alla trasformazione.

Tale processo fu accelerato dalla caduta dell'Impero Romano d'Occidente poichè, spezzata l'unità politica, affievolì quella civile e letteraria.

Il latino subi notevoli trasformazioni oltre che per il fattore suddetto, per le diverse invasioni barbariche e l'influenza di elementi linguistici introdotti dal Cristianesimo.

Vero è che, nonostante gli Eruli, i Goti ed i Longobardi venissero come conquistatori, essi riconoscevano nel latino una lingua superiore. Successsivamente cercarono di impararla inframischiandovi il proprio idioma, dando cosi origine ad un latino profondamente diverso dal classico per suoni, pronunzia e struttura sintattica.

Anche i Franchi ammisero la superiorità della lingua dei vinti. Romanizzandosi, essi contribuirono maggiormente all'avanzare del volgare perchè, come i barbari antecedenti, introdussero voci del loro linguaggio.

II latino passò quindi per gradazioni svariatissime, dando origine alla lingua italiana, alla francese, alla provenzale, alla spagnola, alla portoghese, alla romena e alla ladina.

Molte furono le trasformazioni subite dal latino, quali l'aggiunta dell'articolo, la perdita dei casi e del genere neutro, 
l'aggiunta del condizionale e, nell'indicativo, del passato prossimo e passato remoto, la preferenza delle forme finite a quelle infinitive etc.

Fra thite le lingue citate, il volgare italiano fu quello che più mantenne l'unità latina, nutrendosi della ricca cultura antica.

Il volgare italiano, oltre che assorbire la grandezza del latino, derivò da un particolare linguaggio: il fiorentino.

Perchè si sia scelto il fiorentino come lingua letteraria, è spiegabile da una serie di fattori, particolarmente per essere tale linguaggio il piú affine al latino.

Non è da escludere la posizione geografica della Toscana, che, situata al centro dell'ltalia, influenzava, molto più che le altre regioni, il nord ed il sud.

Indispensabile è anche ricordare che la letteratura in volgare, sia essa in prosa o in verso, malgrado abbia avuto un breve periodo aureo in Sicilia, alla corte degli Svevi, si radicò in Toscana, brillando con Dante, Petrarca e Boccaccio.

Ci siamo riferiti alla "Carta di Capua", come prima documentazione scritta del volgare. Realmente, sin dal 746, in un documento lucchese s'incontra:

"...de uno latum decorre via pubblica".

All'inizio del sec. IX appartiene l'indovinello veronese:

"Boves se pareba

e albo versirio teneba

alba pratalia areba

e negro semen semineba".

Tuttavia, il vero uso del volgare, ossia il suo uso pubblico con espressioni di una certa crganizzazione sintattíca, si ha nel 960 con la "Carta di Capua".

La "Carta di Capua", semplice pergamena del formato di una lettera, è custodita in una cassetta di quercia, nel Monastero di Monte Cassino. 


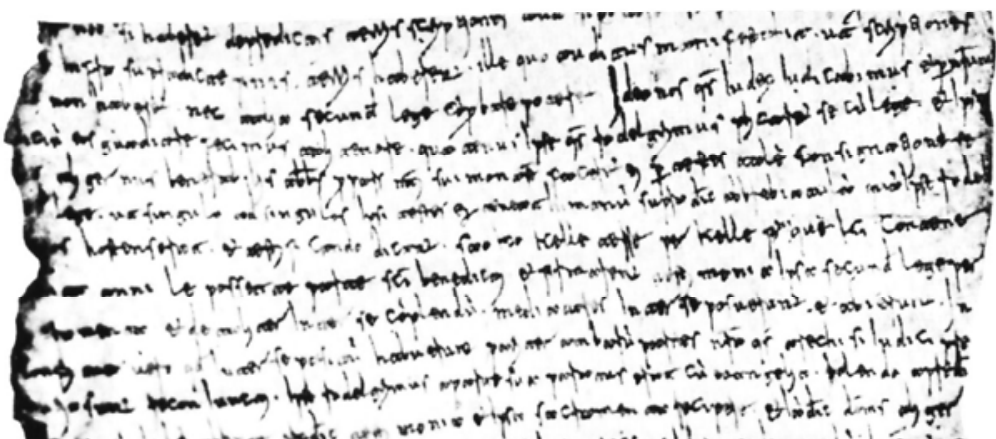

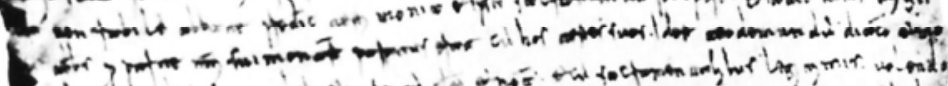

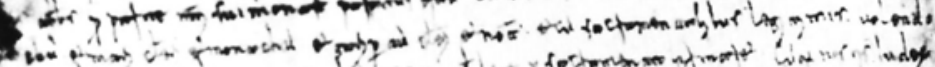

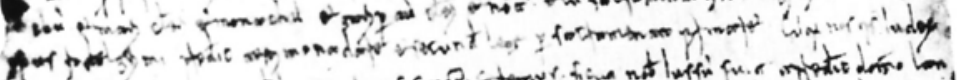

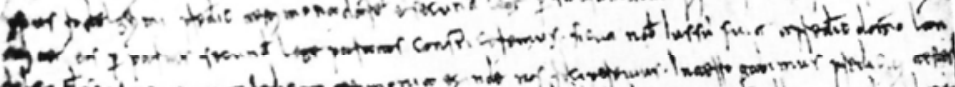

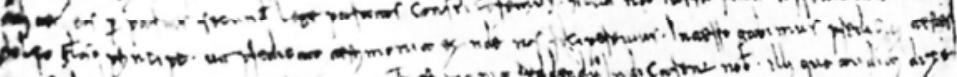

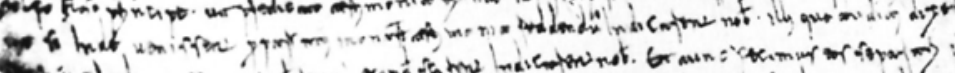

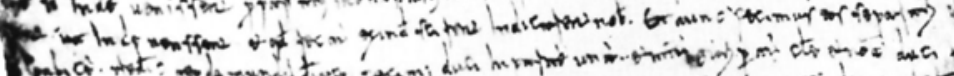

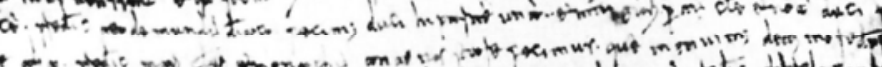

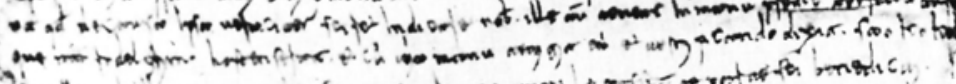

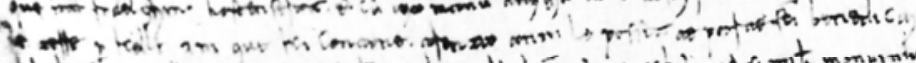

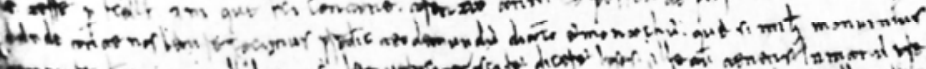

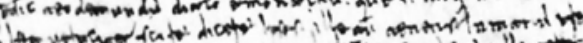
(a) a

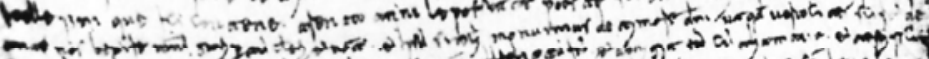

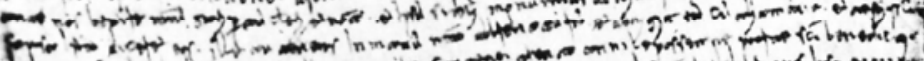

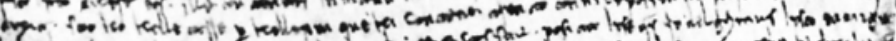

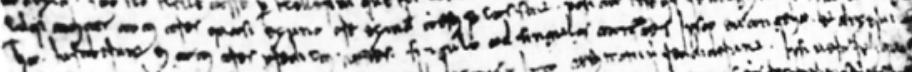

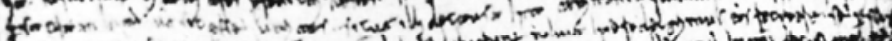

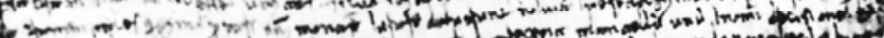

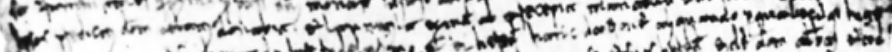

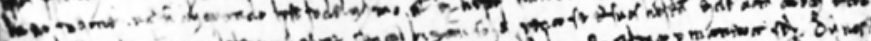

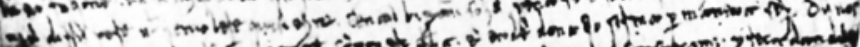

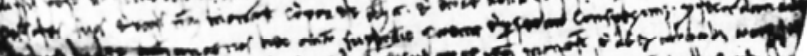

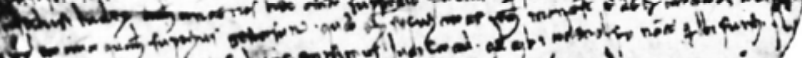

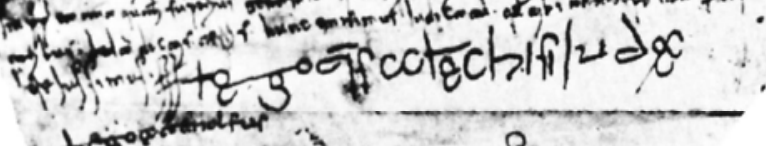

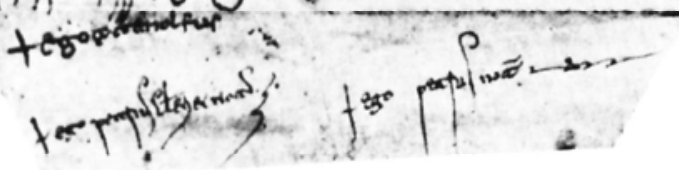

"A CARTA DE CÁPUA",

março de 960 
Quest'anno, solenni commemorazioni millenarie vengono celebrate in Italia per ricordare la famosa "Carta di Capua", in cui $i$ linguisti vedono il primo e chiaro documento della Lingua Italiana.

L'inizio, quindi, di una lingua neolatina che, attraverso lunghe vicende linguistiche, sarebbe diventata lo strumento di espressione del pensiero "di uno dei più famosi popoli della terra".

Poeti e letterati, attraverso i secoli, erano destinati a dare grande bellezza formale alla lingua italiana, che, pen lungo tempo, fu considerata con certo disprezzo dai dotti, i quali preferivano scrivere le loro opere in latino. Ma già Dante Alighieri aveva, col suo genio, intravisto il glorioso e certo trionfo del volgare italiano quando lo definiva con amorosa passione nel suo

\section{Convivio:}

"Luce nuova, sole nuovo, lo quale surgerà là dove d'usato tramonterà, e darà lume a coloro che sono in tenebre e in oscuritate per lo usato sole che a loro non luce" (3).

(3) Dante Alighieri, Convivio, t. I, C. VIII, 12. 